\title{
Squamous Cell Carcinoma of the Breast in a Patient with HIV : A Case Report
}

\author{
Fumi Saito ${ }^{1)}$, Hideaki Ogata ${ }^{1)}$, Tetsuo Nemoto2), Chikako Hasegawa2), \\ Shunsuke Magoshi ${ }^{1)}$, Shinsaku Kanazawa ${ }^{1)}$, Yorichika Kubota ${ }^{1)}$ and Hironori Kaneko ${ }^{1)}$ \\ ${ }^{1)}$ Division of General and Gastroenterological Surgery, Department of Surgery (Omori), \\ Toho University School of Medicine, \\ ${ }^{2)}$ Department of Surgical Pathology, Toho Unversity School of Medicine
}

\begin{abstract}
A 70-year-old woman receiving highly active antiretroviral therapy (HAART) for human immunodeficiency virus (HIV) infection presented at our clinic after she noticed a 1-cm mass in her left breast. A palpable tumor was diagnosed as squamous cell carcinoma with fine needle biopsy, but imaging revealed that the main lesion was surrounded by multiple tumors. We performed a left total mastectomy. Histopathology of the surgical specimen revealed an invasive squamous cell carcinoma surrounded by multiple ductal adenomas. There are few reports of neoplastic lesions in the breasts of patients with HIV. We report an illustrative case and discuss our findings in light of recent reports.
\end{abstract}

Key words: squamous cell carcinoma, HIV, breast cancer

\section{Introduction}

Highly active antiretroviral therapy (HAART) was introduced in 1996 and has significantly improved prognosis for individuals with human immunodeficiency virus (HIV). By suppressing viral activity and maintaining a low viral burden, it is possible to return the patient to $\mathrm{CD} 4$ level, thus significantly

Received: November 22, 2011/Accepted: February 6, 2012

Correspondence to: Hideaki Ogata

Division of General and Gastroenterological Surgery, Department of Surgery (Omori), Toho University School of Medicine, 6-11-1 Omori-Nishi, Ota-ku, Tokyo 143-8541 Japan reducing AIDS deaths and the development of AIDS-related symptoms. However, patients with longstanding HIV infection may develop other problems, including diabetes, kidney and heart problems, and even malignant disease. We report a case of breast cancer in a woman who was receiving HAART for HIV infection.

\section{Case report}

Subject : 70-year-old woman.

Chief complaint : tumor in left breast.

Medical history : hypertension, diabetes, cerebral infarction; approximately 5 years previously, she was infected with HIV during heterosexual intercourse.

Family history : unremarkable.

Present illness : 3 years previously, the patient began receiving HAART at our infectious disease medicine department for treatment of HIV infection. While on HAART, the patient noticed a tumor in her left breast approximately 1 month before she presented for treatment at our department.

Physical examination : an approximately 1-cm-diameter mass was palpated at $2 \mathrm{~cm}$ above the nipple of the left breast. It was elastic, smooth, and had good mobility, without signs on the surface of the skin. Neither axillary nor other regional lymph nodes were palpable.

Laboratory findings : HIV1/2 antibody was positive ; no other abnormalities were noted.

Imaging findings : Mammography revealed many clearly demarcated tumors in the left breast. The characteristic features of malignancy were not seen in a number of the tumors (Fig. 1). Ultrasonography 


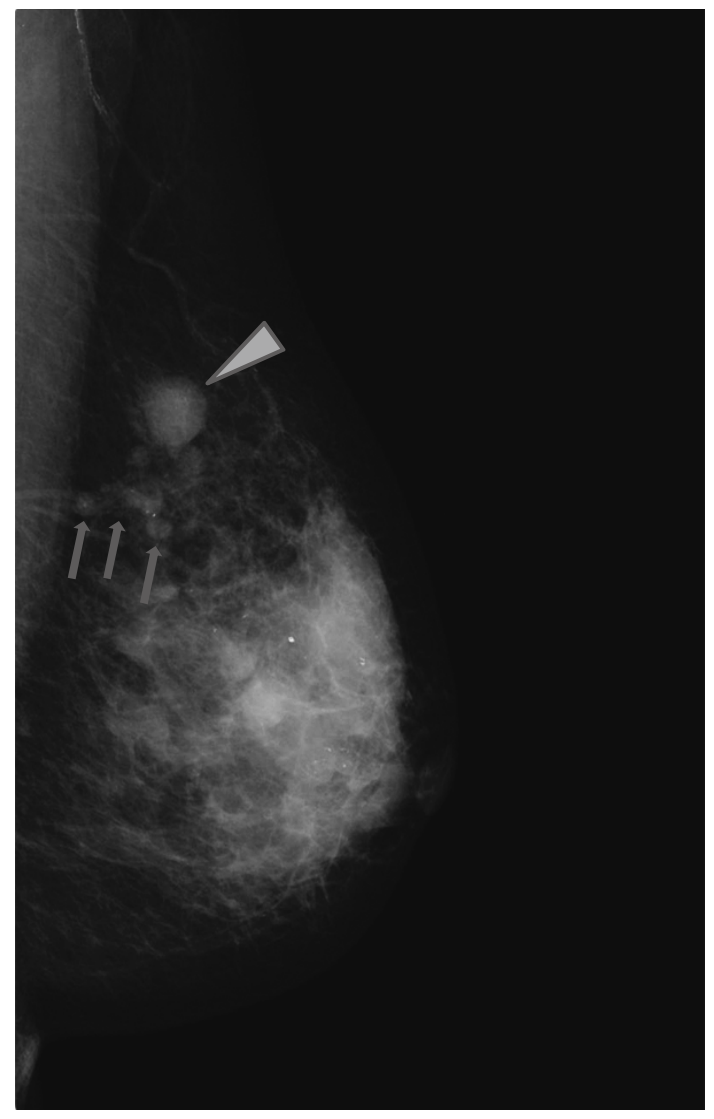

Fig. 1 A mammogram shows the main tumor (arrowhead) surrounded by multiple adjacent tumors (arrows).

revealed that the palpated tumor was irregularly shaped with unclear borders and internal heterogeneity (Fig. 2a). Furthermore, there were multiple adjacent tumors in region $\mathrm{A}$. These tumors were round and had clear borders, and some had heterogeneous internal areas (Fig. 2b). Magnetic response imaging showed findings similar to those from ultrasonography. The main tumor was surrounded by multiple tumors. Contrast enhancement of the main tumor suggested malignancy (Fig. 3 ). Computed tomography and bone scintigraphy revealed no malignancies in any other part of the body.

\section{Cytologic and histopathologic findings}

Fine-needle aspiration cytology of the main tumor revealed small aggregations of large tumor cells with abundant cytoplasm and severely atypical nuclei : these cells appeared to have originated in the malignant tumor and showed squamoid differentiation (Fig. 4). As for the adjacent tumors, three times fine-needle aspiration cytology procedures revealed cells clusters composed of both myoepithelial and epithelial cells, suggesting that bilayered structure was preserved. Apocrine metaplasia was also observed; no malignancies were found.

Needle biopsy of the main tumor revealed invasive carcinoma with irregularly shaped cell nests. The cells with abundant clear cytoplasm were clearly defined and had a cobblestone appearance. Their nuclei were pleomorphic and had enlarged nucleoli. The lesion was diagnosed as squamous cell carcinoma (Fig. 5 ).

Although cytology of the adjacent tumors showed benign findings, the possibility of malignancy could not be completely excluded. We thus performed a total mastectomy.

\section{Histopathology of the surgical specimen}

The largest diameter of the main tumor was $0.9 \mathrm{~cm}$. The pathologic features observed in the needle biopsy were observed in the surgical specimen, confirming a diagnosis of invasive squamous cell carcinoma. The examination did not reveal elements of ductal carcinoma in situ. There was no metastasis to axillary lymph nodes. Estrogen receptor and progesterone receptor were both negative, and the human epidermal receptor-2 score was 0 . The multiple adjacent lesions had clearly defined boundaries surrounded by connective tissue, focal hyalinization and a bilayered structure. The cells displayed apocrine metaplasia in their cytoplasm. These lesions were diagnosed as ductal adenoma (Fig. 6 ). The final diagnosis was Stage I invasive squamous cell of the breast with multifocal ductal adenoma.

We did not perform postoperative chemoendocrine therapy. At this writing, 5 years after surgery, there has been no recurrence of cancer.

\section{Discussion}

Patients with AIDS can develop Kaposi sarcoma, non-Hodgkin lymphomas, primary cerebral lymphomas, and invasive cervical cancer, but breast cancer is not considered to be a related malignant tumor. Few studies have reported cases of HIV-positive patients with breast cancer ; thus, the association of HIV with the pathogenesis and progression of breast 


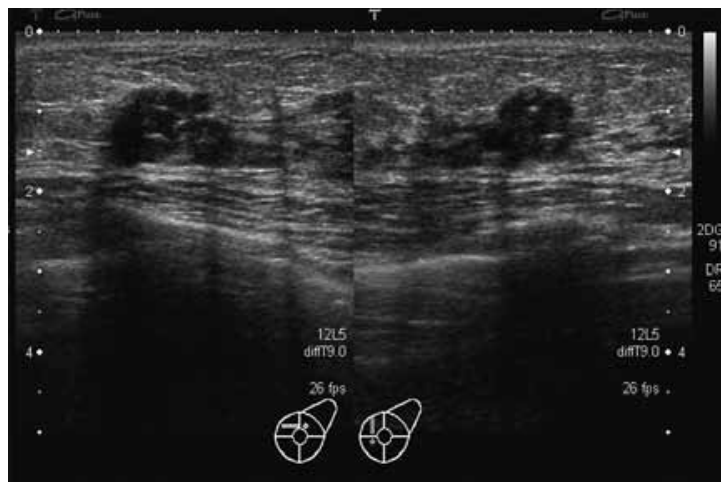

a

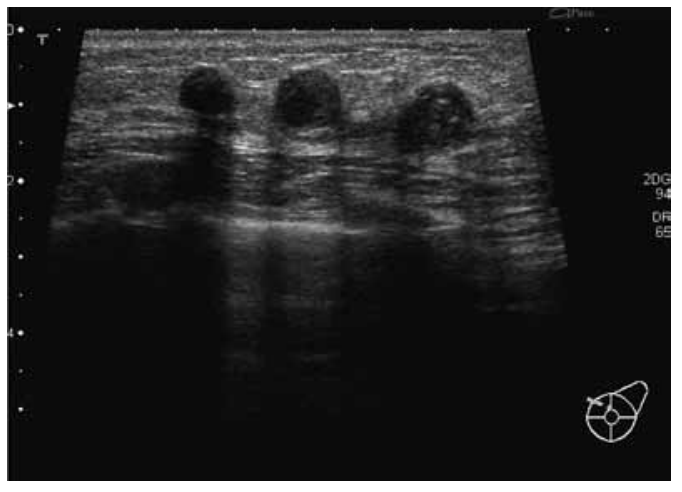

b

Fig. 2 a) An ultrasonogram shows the main tumor detected in the left breast upon palpation, located approximately $2 \mathrm{~cm}$ from the nipple at the 10 o'clock position. The tumor is approximately $1.6 \times 1.2 \mathrm{~cm}$, with an uneven shape and internal heterogeneity.

b) Multiple adjacent tumors are visible in region $A$. Their boundaries are more clearly defined than those of the main tumor, but some internal heterogeneity is visible.

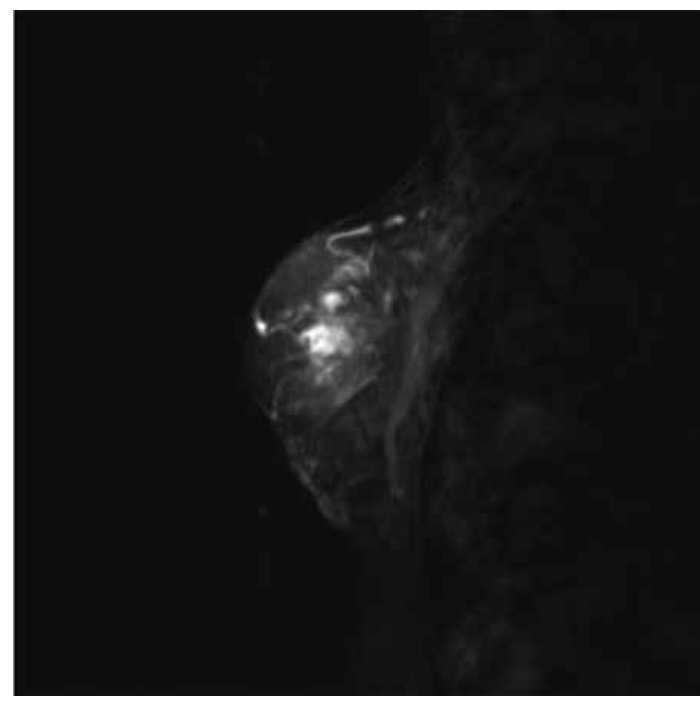

Fig. 3 Magnetic response imaging confirms the presence of the main tumor (arrowhead) and multiple adjacent tumors (arrow).

cancer is unclear. The few available case reports indicate that the prognosis for HIV-positive patients with breast cancer is unfavorable ${ }^{1)}$, as such patients tend to be young and develop recurrences. However, it is unclear whether the unfavorable outcomes are due to general immunosuppression caused by HIV or whether HIV itself attacks the cells, thereby triggering carcinogenesis. On the other hand, it has been suggested that the generally unsatisfactory

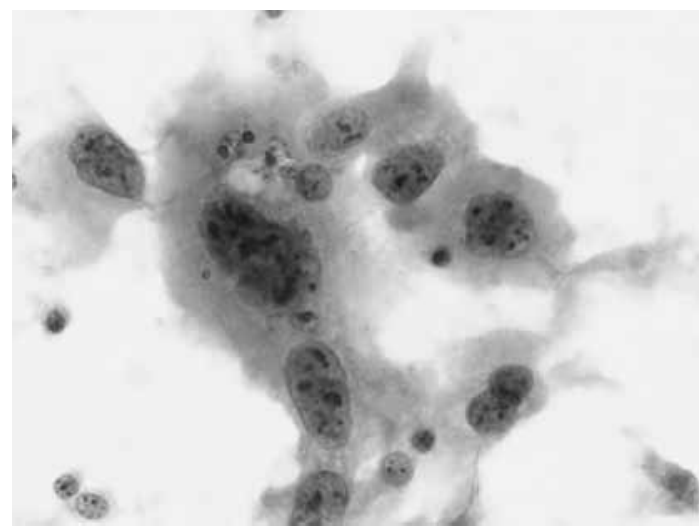

Fig. 4 Fine-needle aspiration cytology of the main tumor shows large tumor cells with abundant cytoplasm and severely deformed nuclei, suggesting breast cancer with squamoid differentiation.

prognosis for breast cancer in HIV-positive patients is due to the fact that the disease is already advanced by the time HIV-positive patients seek treatment ${ }^{2}$. In the United States, for example, there is a higher rate of HIV among the poor and those without medical insurance, and such people are much less likely to seek treatment for medical problems. As a result, late diagnosis and treatment of breast cancer in this population could be largely responsible for their generally poor prognosis.

As for the pathologic characteristics of breast can- 


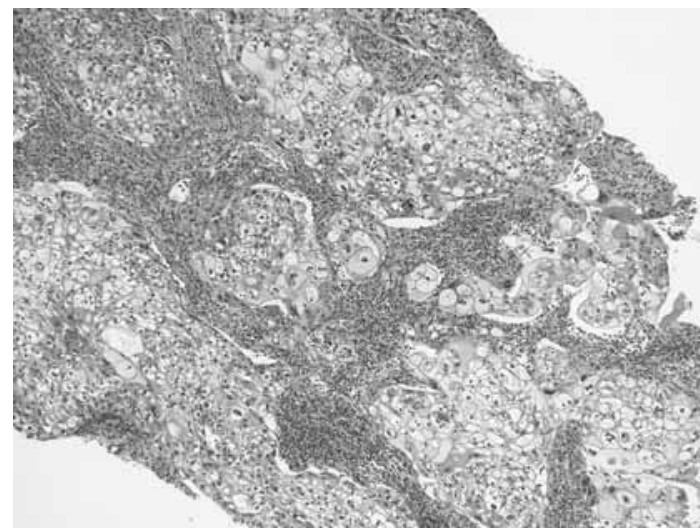

Fig. 5 Needle biopsy shows tumor cells with abundant cytoplasm and pleomorphic nuclei forming cell nests. These cells were clearly defined and had a cobblestone appearance. The lesion was diagnosed as squamous cell carcinoma $(\mathrm{HE} \times 10)$.

cer, HIV-positive patients display a relative abundance of atypical tissue types, including ductal carcinoma accompanied by neuroendocrine cell morphology, mucinous carcinomas with mucin evenly secreted over large areas, and breast cancer with large plasmacytoid cells $\mathrm{s}^{3}$. As for the present patient, her squamous cell carcinoma of ductal origin is rare, accounting for only $0.17 \%$ to $0.4 \%$ of all breast cancers. Indeed, it is classified as a special type in General Rules for Clinical and Pathological Recording of Breast cancer $^{4)}$. However, no reports have claimed that squamous cell carcinoma occurs more often in immunocompromised patients, such as those with HIV, and thus its relationship to HIV is unclear.

Several studies have reported that HIV infection induces degenerative changes of the breast other than malignant lesions. Refractory mastitis and mammary abscesses induced by phlogogenic bacteria such as Salmonella, Pseudomonas aeruginosa, and tubercle bacilli, as well as adiponecrosis and pseudoangiomatous stromal hyperplasia (PASH), can lead to formation of tumors that must be carefully distinguished from breast tumors ${ }^{3}$. One report found that $59 \%$ of breast disease in 46 HIV patients (male and female) was benign, with the following diseases occurring in descending order of frequency : proliferating degeneration and fibrocystic changes, enlargement and swelling of the breast, and fibroadenoma ${ }^{5}$. It has also been reported that breast swelling can de-

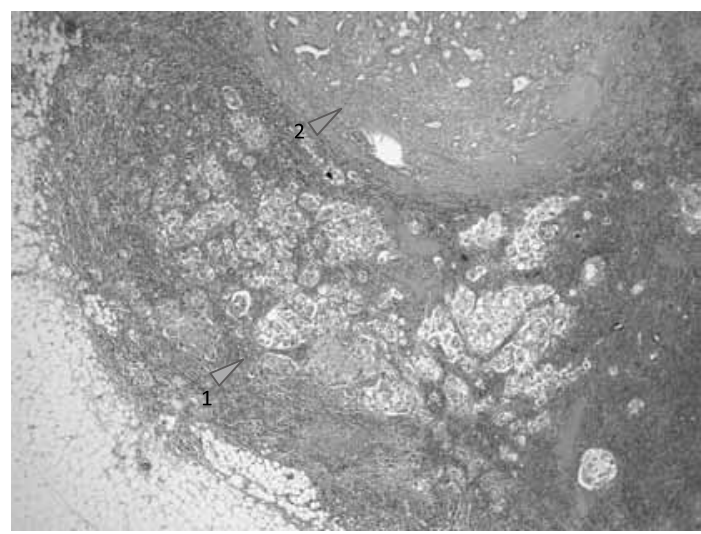

Fig. 6 Pathologic findings of the surgical specimen confirmed a diagnosis of invasive squamous cell carcinoma (arrowhead 1). The multiple adjacent lesions have clearly defined boundaries, focal hyalinization, apocrine metaplastic changes, and a bilayered structure. Ductal adenoma was diagnosed (arrowhead 2) (HE×4).

velop as a symptom of lipodystrophy, which sometimes arises as a side effect of HAART ${ }^{3)}$. It is important to remember that the breast of HIV-positive patients can easily develop infectious, proliferative, and chemically induced degeneration.

Regarding medical treatment, as in breast cancer patients without HIV, patients with HIV are usually treated with standard chemoradiotherapy ${ }^{6-13)}$, and no reports have indicated that combination chemotherapy for breast cancer increases the severity of HIV infection. Many studies have examined simultaneous treatment with HAART and chemotherapy for AIDS-related malignancies, such as non-Hodgkin lymphomas, and have confirmed the efficacy of such combined therapy ${ }^{14)}$. In the present case, anti cancer drugs were not given because the carcinoma was T1N0M0 (Stage I) and because she would not be responsive to hormone therapy. However, early squamous cell carcinoma is associated with a 5 year survival rate of $90 \%{ }^{15}$. It has been 5 years since her mastectomy, and there is no clinical evidence of recurrence or metastasis.

\section{Acknowledgments}

The authors would like to thank Professor Futoshi Akiyama, Division of Pathology, the Cancer Institute of the Japanese Foundation for Cancer Research, for 
his kind advice on the pathologic diagnosis, which was invaluable in the preparation of this paper.

\section{References}

1) Hurley J, Franco S, Gomez-Fernandez C, et al : Breast Cancer and human immunodeficiency virus : a Report of 20cases. Clin Breast Cancer $2: 215-220,2001$

2) Goedert JJ, Schairer C, McNeel TS, et al : Risk of breast, ovary and uterine corpus cancers among 85268 women with AIDS. Br J Cancer 95 : 642-648, 2006

3) Pantanowitz L, Connolly JL : Pathology of the breast associated with HIV/AIDS. Breast J 8 : 234-243, 2002

4) Japan Breast Cancer Society : General Rules for Clinical and Pathological Recording of Breast cancer. The 16th Edition, Kanehara, Tokyo, 2008, P18-30

5) Pantanowitz L, Sen S, Crisi GM, et al : Spectrum of breast disease encountered in HIV-positive patients at community teaching hospital. the Breast $20: 303^{-}$ 308,2011

6) Cuvier C, Espie M, Extra LM, et al : Breast cancer and HIV Infection : Two case Reports. Eur J Cancer 33 : 507-508, 1997

7) Guth AA : Breast Cancer and HIV : What Do We Know? Am surg $65:$ 209-211, 1999

8) Remick SC, Harper GR, Abdullah MA, et al : Metastatic breast cancer in a young patient seropositive for human immunodeficiency virus. J Natl Cancer Inst 83 : 447448, 1991

9) Gachupin-Garcia A, Selwyn PA, Bunder NS : Population-based study of malignancies and HIV infection among injecting drug users in a New York City methadone treatment program, 1985-1991. AIDS 6 : 843-848, 1992

10) Spina M, Nasti G, Simonelli C, et al : Breast cancer in a woman with HIV infection : a case report. Ann Oncol 5 : 661-662, 1994

11) El-Rayes BF, Berenji K, Schuman $P$, et al : Breast cancer in woman with human immunodeficiency virus infection : implications for diagnosis and therapy. Breast cancer Res Treat 76 : 111-116, 2002

12) Soji F, Oluwole : Breast Cancer in a woman with HIV/ AIDS. Jounal of Surgical Oncology $89: 23-27,2005$

13) Oluwole SF, Ali AO, Shafaee $Z$, et al : Breast Cancer in a woman with HIV/AIDS. J Surg Oncol 89 : 23-27, 2005

14) Navaro WH, Kaplan LD : AIDS-related lymphoproliferative disease. Blood $107: 13-20,2006$

15) Kaise H, Sakamoto G, Akiyama F, et al : A clinicopathological study of 22 primary Squamous cell carcinomas of the Breast. Jpn J Breast Cancer 15 : 182-186, 2000 